\title{
Effect of GeCl4/SiCl4 flow ratio on germanium incorporation in MCVD process
}

\begin{abstract}
Germanium and Silica co-deposition in MCVD process is studied with varying the flow ratio of $\mathrm{GeCl} 4 / \mathrm{SiCl} 4$ at high temperature without phosphorus. The range of ratio is from 0.1 to 0.6 with temperature deposition $2100^{\circ} \mathrm{C}$. The incorporation of germanium in silica matrix is deduced from the EPMA SEM-EDX. The experimental result is compared to theory such as thickness deposited layer and mole fraction of germanium in silica.
\end{abstract}

Keyword: MCVD; Thermophoretic; Chloride oxidation; Silica preform; Germanium doped silica; EPMA 